\title{
Adaptive strategies of motile bacteria in dynamic aquatic ecosystems. A simulation study
}

\author{
A.I. Klimenko*, Yu.G. Matushkin, S.A. Lashin \\ Institute of Cytology and Genetics SB RAS, Novosibirsk, Russia \\ Novosibirsk State University, Novosibirsk, Russia \\ *e-mail:klimenko@bionet.nsc.ru
}

Key words: marine bacteria, chemotaxis, motility, energy budgets, adaptive strategies, individual-based modelling

Motivation and Aim: There are two different strategies living organisms resort to in their struggle for existence facing the environmental changes - either adaptation to existing conditions, e. g. by adjusting their metabolism to alternative energy source, or migration following the optimal environmental conditions invading new biotopes where they compete with the species of a local community. Both strategies are relevant for microbial ecosystems and both the mechanisms that drive the emergence of species following different adaptation strategies and the conditions that favor the domination of certain variants are of a fundamental scientific interest. Current estimates show that energy expenditure of various marine bacteria ranges from 2 to $50 \%$ of their total energy budgets [1] with different species varying in motility. However, the causes underlying the sustainability of such a diversity in energy expenditure range remain obscure as its relation to metabolic efficacy does. According to these questions, we have investigated which adaptive strategies emerge during the evolution of traits associated with migration. Methods and Algorithms: We have used the Haploid Evolutionary Constructor 3D (HEC 3D) [2] software complex to build the models of coevolution of metabolic and migratory traits in populations of microorganisms. The HEC 3D allows creating multilayer models of evolution of trophically interlinked populations of motile microorganisms inhabiting spatially structured environments.

Results: Investigating the models of microbial populations characterized by the polymorphism in gene that controls migratory energy costs, we have found out that given homogeneous initial distribution of cells there are two different adaptive strategies: either decreasing energy costs of migration and performing regular adaptive migrations towards nutrient rich biotopes or reluctance in spending energy for migration along with a respective increase in energy costs of motility.

Conclusion: It has been demonstrated that the nutrient variable conditions and homogeneous initial distribution of cells cause the disruptive selection by the trait of migratory energy costs. We suggest such a mechanism to be underlying the corresponding processes that are observed in nature.

Acknowledgements: The study was supported by the budget project 0324-2018-0017.

References:

1. Stocker R., Seymour J.R. (2012). Ecology and physics of bacterial chemotaxis in the ocean. Microbiol. Molecular Biology Reviews. 76(4):792-812.

2. Klimenko A.I. et al. (2015) Modeling evolution of spatially distributed bacterial communities: a simulation with the haploid evolutionary constructor. BMC Evol. Biol. 15:S3. 